This item was submitted to Loughborough's Research Repository by the author.

Items in Figshare are protected by copyright, with all rights reserved, unless otherwise indicated.

\title{
Closely coupled metallodielectric electromagnetic band-gap structures formed by double-layer dipole and tripole arrays
}

PLEASE CITE THE PUBLISHED VERSION

http://dx.doi.org/10.1109/TAP.2004.827530

\section{PUBLISHER}

(C) Institute of Electrical and Electronics Engineers (IEEE)

VERSION

VoR (Version of Record)

\section{LICENCE}

CC BY-NC-ND 4.0

\section{REPOSITORY RECORD}

Feresidis, Alexandros P., George Apostolopoulos, N. Serfas, and J.C. Vardaxoglou. 2019. "Closely Coupled Metallodielectric Electromagnetic Band-gap Structures Formed by Double-layer Dipole and Tripole Arrays". figshare. https://hdl.handle.net/2134/9452. 
This item was submitted to Loughborough's Institutional Repository (https://dspace.lboro.ac.uk/) by the author and is made available under the following Creative Commons Licence conditions.

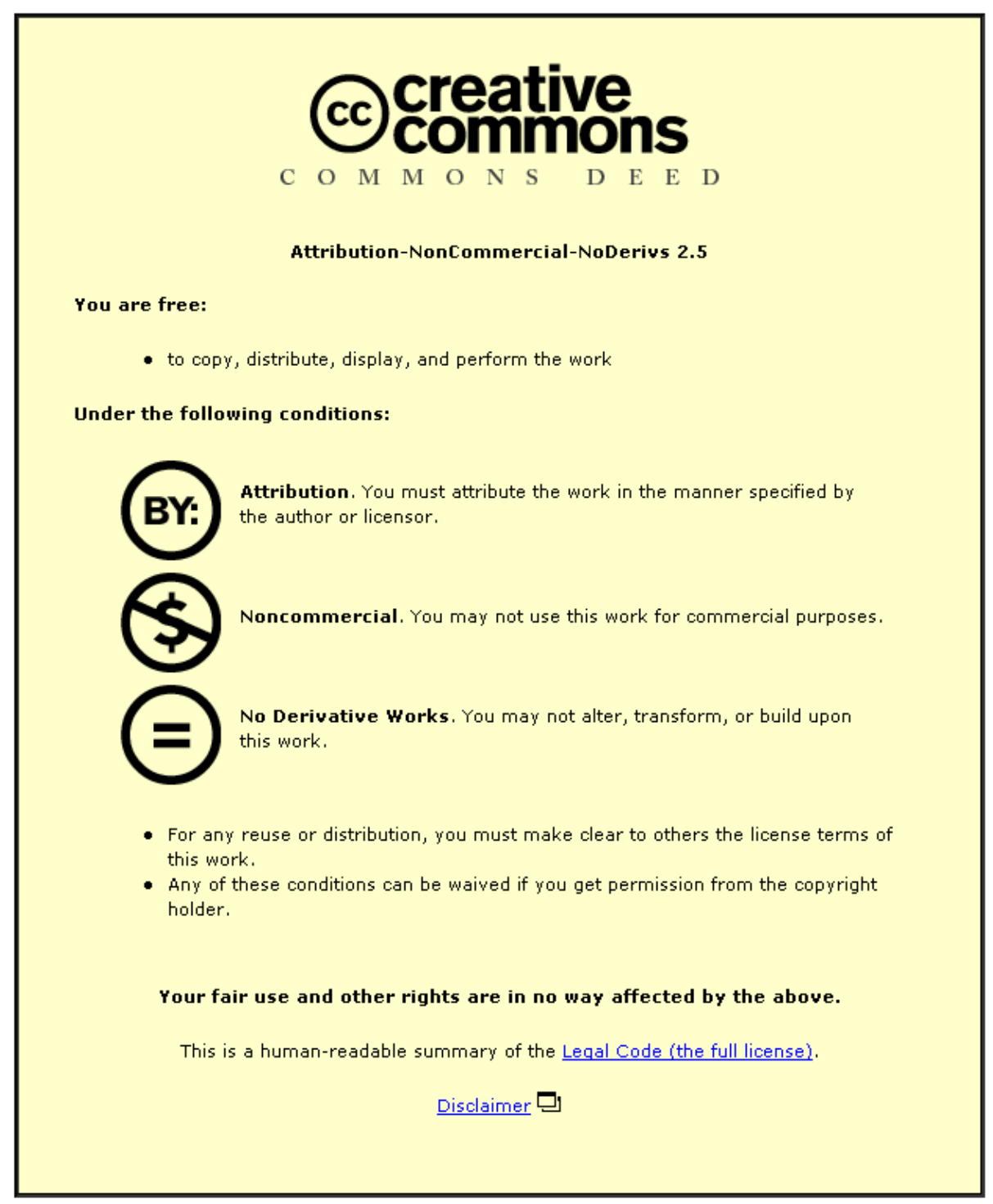

For the full text of this licence, please go to: http://creativecommons.org/licenses/by-nc-nd/2.5/ 


\title{
Closely Coupled Metallodielectric Electromagnetic Band-Gap Structures Formed by Double-Layer Dipole and Tripole Arrays
}

\author{
Alexandros P. Feresidis, Member, IEEE, George Apostolopoulos, Student Member, IEEE, Nikolaos Serfas, and
} John C. Vardaxoglou, Member, IEEE

\begin{abstract}
The concept of closely coupled metallodielectric electromagnetic band-gap (CCMEBG) structures is introduced and investigated using two-dimensional (2-D) double-layer dipole and tripole arrays. An efficient numerical method based on a set of coupled integral equations is used to simulate the double-layer array response. The arrays are placed in close proximity to each other and shifted appropriately in order to produce maximum element coupling. Measurements are presented for oblique plane wave and surface wave incidences. A substantial decrease of the stopband center frequency is observed with the CCMEBG design for both element geometries. Furthermore, wider bandwidth and improved angular stability as compared to single-layer MEBG is obtained. The tripole arrays arranged on a hexagonal lattice exhibit common stopband for any polarization of the incident field due to the symmetry of the element in conjunction with the lattice. The lowering of the resonance for up to 4 to 1 in simulation results emerges as the layers are separated by less than $\lambda / 1200(0.1 \mathrm{~mm}$ at $2.5 \mathrm{GHz})$.
\end{abstract}

Index Terms-Antennas, arrays, electromagnetic band-gap (EBG) structures, frequency selective surfaces, periodic structures.

\section{INTRODUCTION}

$\mathbf{E}$ LECTROMAGNETIC BAND-GAP (EBG) structured materials are a class of artificial periodic metamaterials that prohibit propagation of electromagnetic waves within a particular frequency band. They have emerged as a direct analog to photonic band-gap (PBG) materials used in the optical regime [1], [2], and have been investigated extensively in recent years with regard to applications in the microwave frequency range [3]. EBG materials composed of periodic metallic elements have been studied [4]-[8] as an alternative to photonic crystals formed by inhomogeneities in a dielectric host medium. Resonant periodic arrays of metallic dipoles and tripoles on low loss dielectric substrate have been recently investigated as EBG filters for microstrip patch antennas and printed circuit applications [9]. This class of metallodielectric EBG (MEBG) structures is compatible with printed circuit technology and could potentially be developed into conformal EBG designs using flexible dielectric substrates. Furthermore, the resonant nature of MEBGs makes them prime candidates

Manuscript received October 6, 2002; revised July 3, 2003. This work was supported by a U.K. EPSRC Research Grant GR/R42580/01

The authors are with the Wireless Communications Research Group, Department of Electronic and Electrical Engineering, Loughborough University, Loughborough, LE11 3TU, U.K. (e-mail: A. Feresidis@lboro.ac.uk; J.C.Vardaxoglou@lboro.ac.uk).

Digital Object Identifier 10.1109/TAP.2004.827530 for yielding controlled EBG. For practical applications, they would be required to be small and compact, and exhibit a stable band-gap performance with different plane wave or surface wave incident orientations.

Lowering the resonant frequency enhances the band-gap stability by virtue of avoiding the grating responses. Methods such as capacitive loading of dipole and cross dipole elements and super dense surfaces have been presented [10], [11], but substantial lowering of the resonance could be achieved by close coupling and relative shifting of a double-layer MEBG structure. This will potentially have a beneficial impact on the design of high performance EBGs, because it deals with a size reduction process in an efficient way. Closely coupled arrays have been presented using conducting dipole and slot arrays, as well as a combination of complementary elements [12]-[14]. In general, they are formed by two arrays closely spaced together and shifted appropriately in order to produce high element coupling. Due to the close proximity and the strong coupling between the shifted elements, the effective electrical length of the element increases, resulting in a significant decrease of the array resonant frequency. In an equivalent circuit model of the structure, the high capacitance between elements on different planes increases significantly the overall capacitance thus resulting in the decrease of the resonant frequency. This property is attractive for reducing the size of the structure whilst maintaining superior bandwidth and angular stability and is investigated here in the context of MEBG structures, leading to closely coupled MEBG (CCMEBG) structures.

The concept of CCMEBG structures is general and can be applied to various element geometries. In this paper, we study CCMEBG surfaces formed by double-layer dipole and tripole arrays, whereby the array elements are etched on either side of a thin dielectric substrate. The CCMEBG response is studied for normal and oblique plane wave incidence on the array as well as for surface wave propagation along the array. The transition from plane wave incidence to surface wave propagation is demonstrated and the advantages of the CCMEBG design compared to the single-layer MEBG are shown.

A brief description of the method used to simulate and design the CCMEBG structures is given in Section II. It is an infinite array plane wave modal analysis based on Floquet's theorem and combined with a method of moments solution of a set of coupled integral equations. The key attribute of this method is that it is computationally efficient and exhibits a good relative convergence for small separation distances between the arrays. 


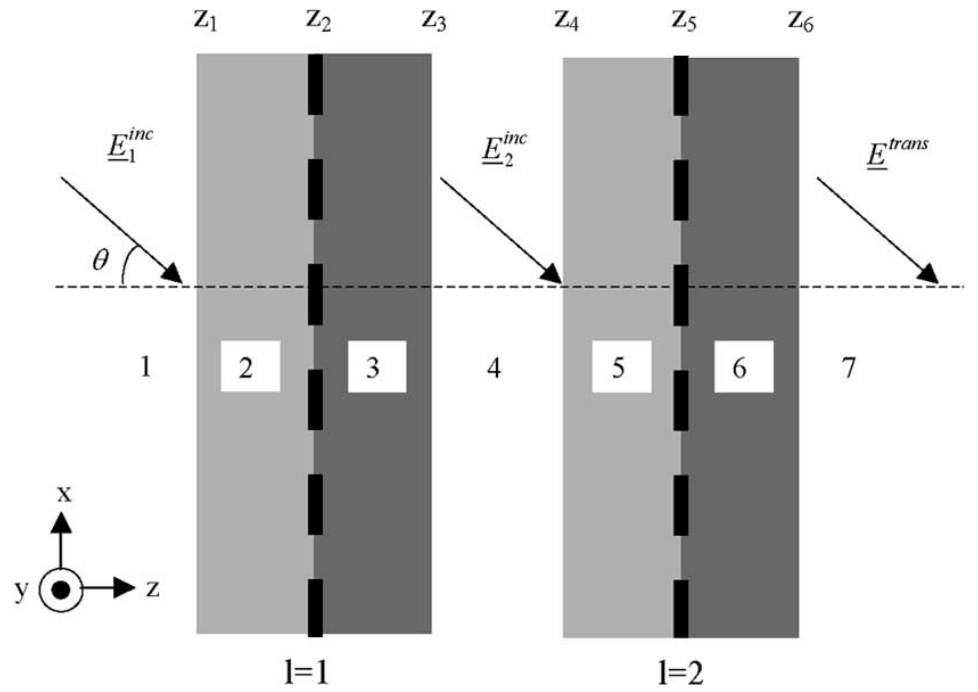

Fig. 1. Geometry of double-layer array structure.

In Section III, dipole arrays are used to form a CCMEBG structure. In Section IV the performance of tripole CCMEBG structures is presented. Tripole arrays on a hexagonal lattice geometry result in a smaller angle of the first irreducible Brillouin zone on the reciprocal lattice and can be used in CCMEBG designs yielding common band gap for any polarization of the incident field. While dipole arrays are unidirectional in the band gap they can be made more compact and exhibit bigger frequency shifts of the resonance.

\section{ANalysis of Closely Coupled ARrays of CONDUCTING ELEMENTS}

We have used a plane wave modal analysis method to simulate the response of closely coupled double-layer arrays [15], [16]. Following this method, a set of coupled integral equations is derived and solved using the method of moments [17]. Other approaches to the analysis of multilayer array structures have been reported, whereby each layer is considered as a separate block and cascaded with others by means of either multiple port circuit techniques [18], [19] or a generalized scattering matrix formulation based on a multimodal equivalent circuit [20]. However, computational constraints are posed when closely spaced arrays are considered in these methods, because they involve manipulation of matrices whose dimensions depend on the Floquet mode number. The coupled integral equation (CIE) method on the other hand is based on the solution of a system of equations with intrinsic higher orders of interaction and is amenable to predicting responses of closely coupled surfaces without excessive computational demands. The major points of the CIE analysis are presented here for the general case of a double-layer array of conducting elements with each array sandwiched between dielectric layers.

Fig. 1 shows the geometry of a double-layer array structure with dielectric support. We assume that the structure consists of infinite periodic arrays with elements of infinitesimal thickness excited by a plane wave, incident at arbitrary angle. The Floquet theorem is employed to simplify the analysis by expanding the tangential scattered fields as well as the induced currents in a

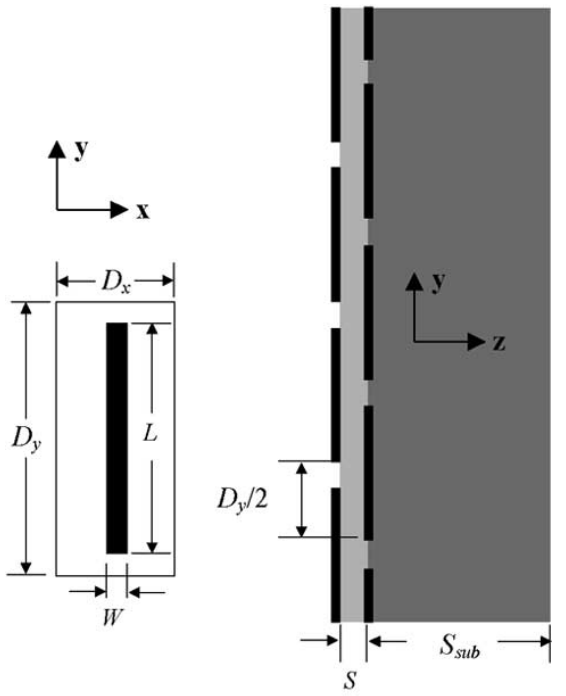

(a)

(b)

Fig. 2. (a) Geometry of dipole array unit cel. (b) Cross section of dipole CCMEBG.

unit cell in terms of Floquet modes $\left(\Psi_{p q}\right)$. The same number of Floquet modes ( $p$ and $q$ ) on each layer and identical array lattices are also assumed. The integral equation defined at the $l$ th boundary $(l=1,2)$ can be obtained by applying the standard electromagnetic boundary conditions at each interface. It is generally written in terms of Floquet modes and the product of the spectrum of the dyadic Green's function, $\tilde{G}$, with the spectrum of the surface currents $\underline{\tilde{J}}^{r^{\prime}}$ where $r^{\prime}=1,2$

$$
\sum_{p q}\left\{\sum_{r^{\prime}=1}^{M} \tilde{G}_{l}^{r^{\prime}} \underline{\tilde{J}}^{r^{\prime}}\right\} \Psi_{p q}=\underline{E}_{l}^{\mathrm{inc}}, \quad l=1,2
$$

where the tangential field, of amplitude $T_{m}^{\text {inc }}$, incident at that locality is

$$
\underline{E}_{l}^{\mathrm{inc}}=2 \sum_{m=1}^{2}\left(\prod_{i=1}^{3 l-1} \tau_{i}\right) \eta_{\mathrm{moo}}^{1} T_{m}^{\mathrm{inc}} \Psi_{o o} \underline{\mathrm{K}}_{\mathrm{moo}}
$$




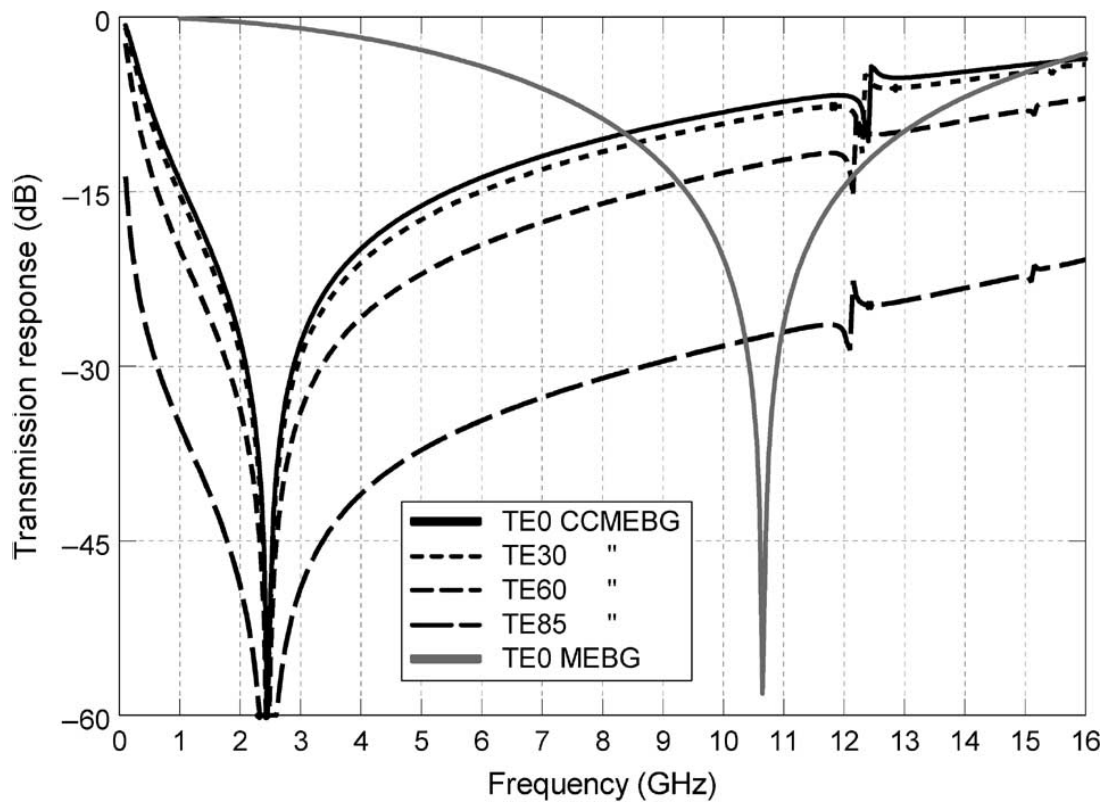

Fig. 3. Simulated transmission response of dipole CCMEBG for different angles of TE plane wave incidence and dipole MEBG for normal incidence.

$\tau$ and $\eta$ are terms involving the modal reflection coefficients and admittance respectively, of the array and the dielectric support at the region concerned. In (2) the modal order is zero and the summation over $m$ denotes the TM $(m=1)$ and TE $(m=2)$ waves. Equation (1) is in coupled form, so the higher order and/or evanescent mode coupling between the two arrays is inherently included. The spectral Green's functions, including the occurrence of the dielectrics, are of the following form

$$
\begin{aligned}
& \tilde{G}_{l}^{r^{\prime}}=\frac{1}{\left(k_{t x}\right)^{2}+\left(k_{t y}\right)^{2}} \\
& \times\left[\begin{array}{cc}
\xi_{l}^{1, r^{\prime}}\left(k_{t x}\right)^{2}+\xi_{l}^{2, r^{\prime}}\left(k_{t y}\right)^{2} & -k_{t x} k_{t y}\left(\xi_{l}^{2, r^{\prime}}-\xi_{l}^{1, r^{\prime}}\right) \\
-k_{t x} k_{t y}\left(\xi_{l}^{2, r^{\prime}}-\xi_{l}^{1, r^{\prime}}\right) & \xi_{l}^{2, r^{\prime}}\left(k_{t x}\right)^{2}+\xi_{l}^{1, r^{\prime}}\left(k_{t y}\right)^{2}
\end{array}\right]
\end{aligned}
$$

$k_{t x}$ and $k_{t y}$ are the Floquet phasor harmonics and $\xi$ are terms involving the dielectric superstrates/substrates, as well as the distance separating the layers (see [15]). Using the method of moments, (1) is reduced into a matrix form by expanding the unknown currents as a set of entire domain basis functions [16]. Once the currents have been estimated the transmitted field can be written for the propagating zero-order Floquet mode $(p=$ $0, q=0)$.

$$
\underline{E}^{+}\left(\underline{r}, z_{6}\right)=\left(\underline{E}_{2}^{\mathrm{inc}}-\sum_{r^{\prime}=1}^{2} \tilde{G}_{2}^{r^{\prime}} \underline{\tilde{J}}^{r^{\prime}}\right) \tau_{6} e^{+j \beta_{p q}^{6} z_{6}} \Psi_{00}(\underline{r})
$$

The transmission coefficient can be calculated from (2) and (4), [15]. The CIE method, as has been mentioned, is computationally efficient with good relative convergence at very close separation distances. Using the same analysis, the single-layer case can be reproduced exactly by taking the separation distance to zero. The dispersion characteristics of the structures could be extrapolated by solving the homogenous version of (1). These are indeed useful for illustration purposes as a qualitative measure [8]. For accurate quantitative characterization one would need a set of forward transmission coefficient results as the angle of incidence and polarization varies. In the following sections a rigorous experimental validation of the CCMEBG concept and the transmission responses are shown in a wide frequency range, and the performance outside the band gap is also presented.

\section{Dipole CCMEBG StRUCTURES}

Dipole arrays are initially utilized to implement a CCMEBG design. The thin linear dipole is the simplest among a variety of elements and it can be used to obtain one dimensional EBG performance. The stopband appears due to the element resonance when the electric field orientation is parallel to the dipole and diminishes as the electric field becomes perpendicular to the dipole (no resonance). The permormance of dipole CCMEBGs is presented and compared to a single-layer MEBG with identical geometry for TE incidence (with respect to $\mathrm{z}$ ). TM plane wave incidence has produced similar effect, and for surface wave incidence only weak TM modes have been excited owing to the configuration used in the measurements.

The geometry of the unit cell of a dipole array is shown in Fig. 2(a). The dipole array geometry studied in [9] has been used, whereby the dipoles are arranged in a square lattice and have the following dimensions: $L=15.5 \mathrm{~mm}, W=0.5 \mathrm{~mm}$, $D_{x}=5 \mathrm{~mm}, D_{y}=17.5 \mathrm{~mm}$. A double-layer structure is formed by combining two identical dipole arrays The second layer is shifted along the $y$-direction with respect to the first one by $D_{y} / 2$ [Fig. 2(b)]. This displacement has been reported [12] to produce maximum aperture coupling between the two layers, thus introducing a maximum shift to the array resonance toward lower frequencies. The two layers are separated by a thin dielectric layer with permittivity $\varepsilon_{r}=3$. A dielectric substrate of thickness $S_{\mathrm{sub}}=1.13 \mathrm{~mm}$ is used to support the arrays. 
(a)

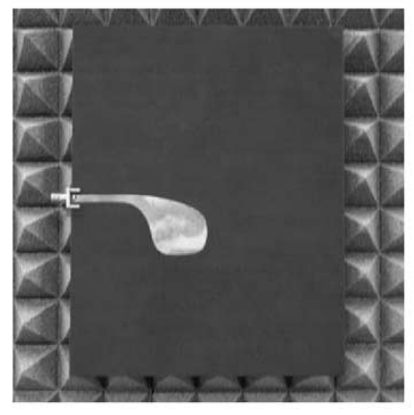

Top

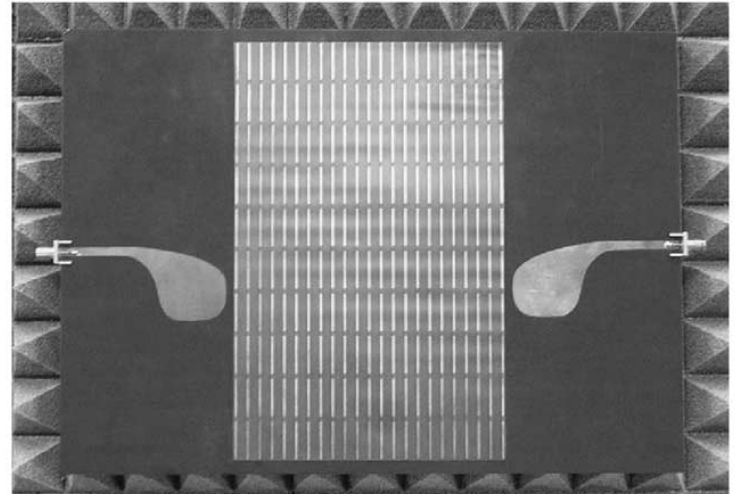

Fig. 4. (a) Top and bottom view (photograph) of balanced Vivaldi antenna. (b) Photograph of surface wave (SW) measurements configuration with dipole CCMEBG.

\section{A. Simulations}

The plane wave modal analysis discussed in Section II is implemented in order to simulate the transmission response of double as well as single-layer dipole arrays. At normal incidence $\left(\theta=0^{\circ}\right)$, with the electric field parallel to the dipoles, the single-layer dipole array resonates at $10.7 \mathrm{GHz}$ and a grating lobe appears at about $17 \mathrm{GHz}$ due to the array $y$-periodicity (Fig. 3). At higher angles of incidence the resonance moves toward lower frequency values $\left(10 \mathrm{GHz}\right.$ for $\left.\theta=85^{\circ}\right)$ and is affected by grating responses, which start appearing at frequencies near and below the resonance.

Simulations have shown that as the array separation distance $S$ is reduced, the coupling between the two layers becomes stronger, resulting in a decrease of the resonant frequency. For $S=0.1 \mathrm{~mm}$ the resonance decreases dramatically and appears at $2.4 \mathrm{GHz}$, which corresponds to more than 4:1 frequency shift. Thus, an electrical dipole length which produces a $\lambda / 2$ resonance is formed due to the interlayer coupling, whereas the physical dipole length in the array is of the order of $\lambda / 8$. Smaller separation distances with values approaching zero would result in a further shift of the resonant frequency, however they become impractical in terms of fabrication and are not presented here. For $S=0 \mathrm{~mm}$, the dipoles of the two arrays would collapse with each other and an inductive grid of infinitely long metallic strips would be formed. The double-layer array with $S=0.1 \mathrm{~mm}$ will be referred to throughout the paper as dipole CCMEBG. Fig. 3 shows the transmission coefficient of the dipole CCMEBG (with $S=0.1 \mathrm{~mm}$ ) for various angles of incidence (TE). The resonance in the CCMEBG design has shifted away from the grating lobe region and is extremely stable with angle of incidence. In addition, a significant increase in the bandwidth of the stopband is observed. To quantify this, the fractional $-15 \mathrm{~dB}$ bandwidth at normal incidence is calculated. In the single-layer case it is $2.5 \mathrm{GHz} / 10.7 \mathrm{GHz}=23 \%$, whereas the dipole CCMEBG exhibits a wider bandwidth of $4.4 \mathrm{GHz} / 2.4 \mathrm{GHz}=183 \%$.

\section{B. Measurements}

The dipole CCMEBG has been fabricated as a $20 \mathrm{~cm}$ square sheet and has been attached on a flat rigid dielectric board $\left(\varepsilon_{\text {rsub }}=2.2, S_{\text {sub }}=1.13 \mathrm{~mm}\right.$ ) of the same size. The thin dielectric layer between the two arrays in the CCMEBG was measured $0.15 \pm 0.02 \mathrm{~mm}$. This means that the separation distance between the two arrays is slightly higher than the simulated and thus the measured resonant frequency is expected a bit higher. In order to carry out plane wave and surface wave measurements over a wide range of frequencies two wideband end-fire antipodal Vivaldi antennas have been used [21]. The antenna is printed on the same dielectric material which is used as a substrate for the CCMEBG. Fig. 4(a) depicts a photograph of both sides of the Vivaldi antenna. The feeding of the antenna is by a smooth transition from a microstrip line to a parallel strip line and then to a symmetrically flared out slot line, which is the antenna element itself. The radiating part is the inner edge of the antenna which is exponentially curved out. Therefore, different locations of the antenna inner edge radiate at different frequencies. Due to the small dielectric thickness the polarization of the radiated field is mainly parallel to the plane of the antenna/dielectric (TE mode). The cross polar component (TM mode) is low at low frequencies and increases at higher frequencies due to the inclination of the radiated electric field.

For the plane wave measurements (normal and oblique incidence), the dipole CCMEBG surface has been placed in an open window of the same dimensions within an absorbing screen. The Vivaldi antennas used as transmitter and receiver, were placed on either side of the CCMEBG array at a distance such that the array was within their far field at frequencies under consideration. By rotating the CCMEBG plane we have measured the transmission response of the structure for various angles of TE incidence. For comparison purposes, the same set of measurements was carried out for a single-layer MEBG with exactly the same array dimensions. The limited gain of the Vivaldi antennas, which was measured between 6 and $3 \mathrm{~dB}$ over the operating frequency range, restricted the dynamic range of the measurements. In addition, at low frequencies the size of the CCMEBG was relatively small $(2 \lambda$ at $3 \mathrm{GHz})$ which altered the transmission response of the array compared to the one predicted from the infinite array analysis in Section II. For these reasons the simulation results are not compared with measurements here and they are just used as an indication of the expected CCMEBG response.

The dipole CCMEBG response for surface wave propagation along the array with the electric field parallel to the element orientation has also been measured. In these measurements the forward surface wave transmission response is taken on the plane 
(a)

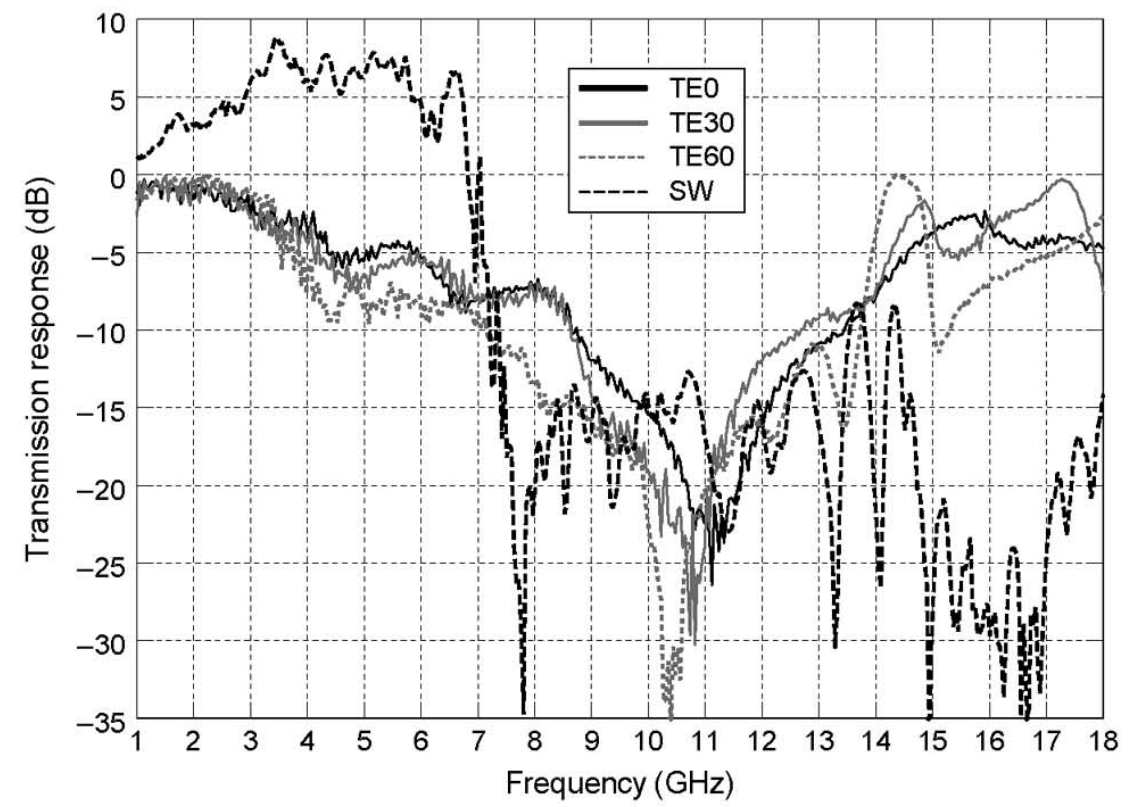

(b)

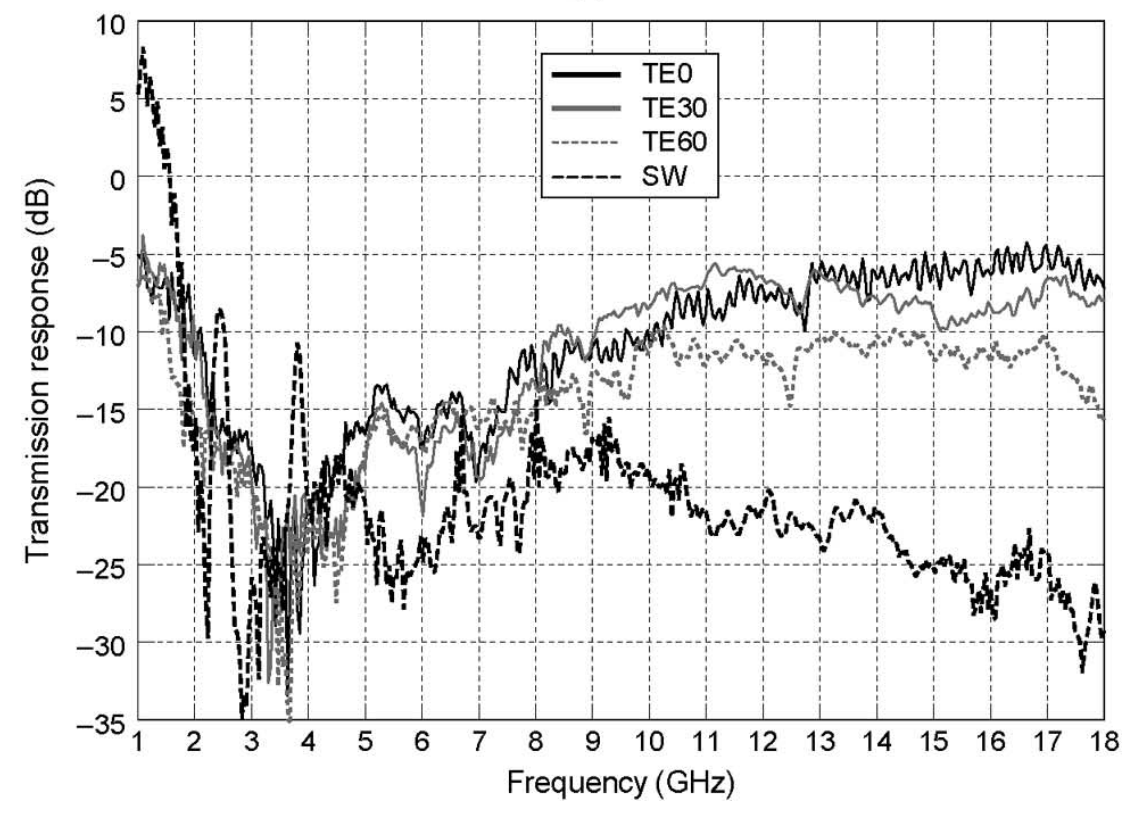

Fig. 5. Measured transmission response of (a) single-layer dipole MEBG. (b) Dipole CCMEBG for TE plane wave and surface wave (SW) incidences.

of and normalized with respect to the Vivaldi antennas alone. The transmitting and receiving Vivaldi antennas are printed on the same dielectric slab. This could be considered equivalent to a $90^{\circ} \mathrm{TE}$ incidence but the wave is now bound to the surface. The configuration used for this measurement is shown in the photo of Fig. 4(b). A dipole CCMEBG of smaller size is placed between the antennas. This configuration excites mainly TE modes along the array, however the cross polar components of the Vivaldi antennas cause a weak excitation of TM modes.

The plane wave and surface wave responses of the dipole CCMEBG and the single-layer dipole MEBG are shown in Fig. 5. The ripple at normal incidence is due to standing waves. A study of the transmission responses confirms the theoretical predictions. The resonant frequency at normal incidence moves from $11 \mathrm{GHz}$ (MEBG) to about $3.5 \mathrm{GHz}$ (CCMEBG), which corresponds to more than 3:1 shift. Simulation results with separation $S=0.17 \mathrm{~mm}$ have reproduced accurately the measured resonant frequency. Due to the fact that the CCMEBG resonant frequency is well below the grating lobe frequencies, the CCMEBG response is less sensitive to the angle of incidence than the single-layer MEBG. Furthermore, the CCMEBG exhibits a wider band gap. The $-10 \mathrm{~dB}$ fractional bandwidth for the CCMEBG at normal incidence is $98 \%(5.4 \mathrm{GHz}$ absolute bandwidth) compared to $42 \%$ (4.6 GHz absolute bandwidth) for the MEBG.

The surface wave transmission response has shifted accordingly with the plane wave response. Both MEBG and CCMEBG surface wave responses yield a gain of up to $8 \mathrm{~dB}$ before the 


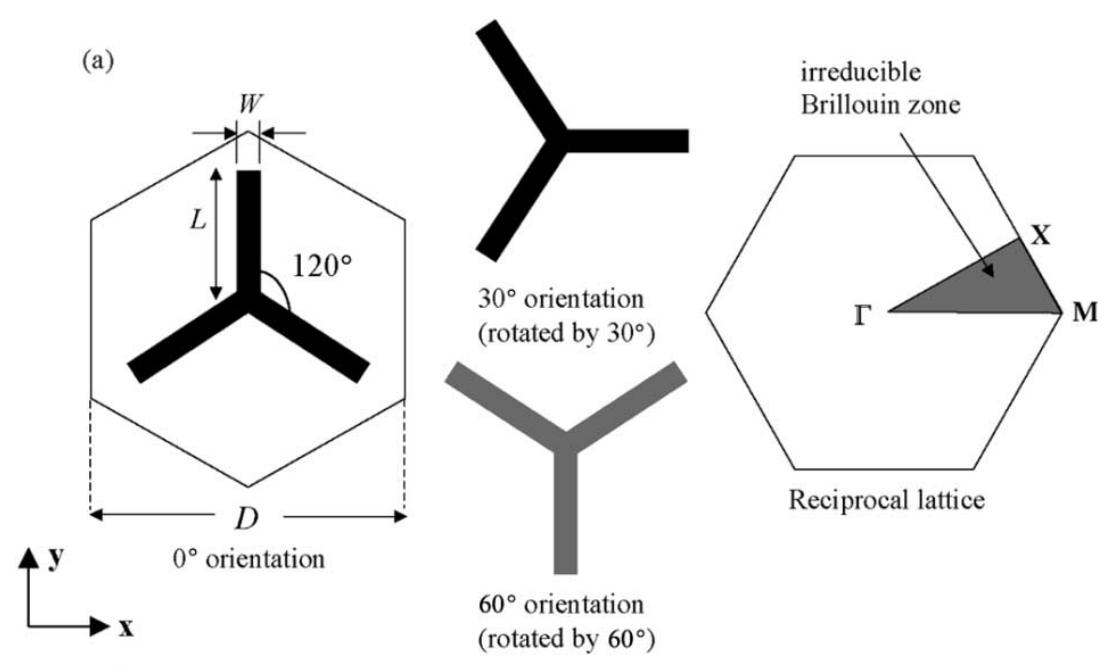

(b)

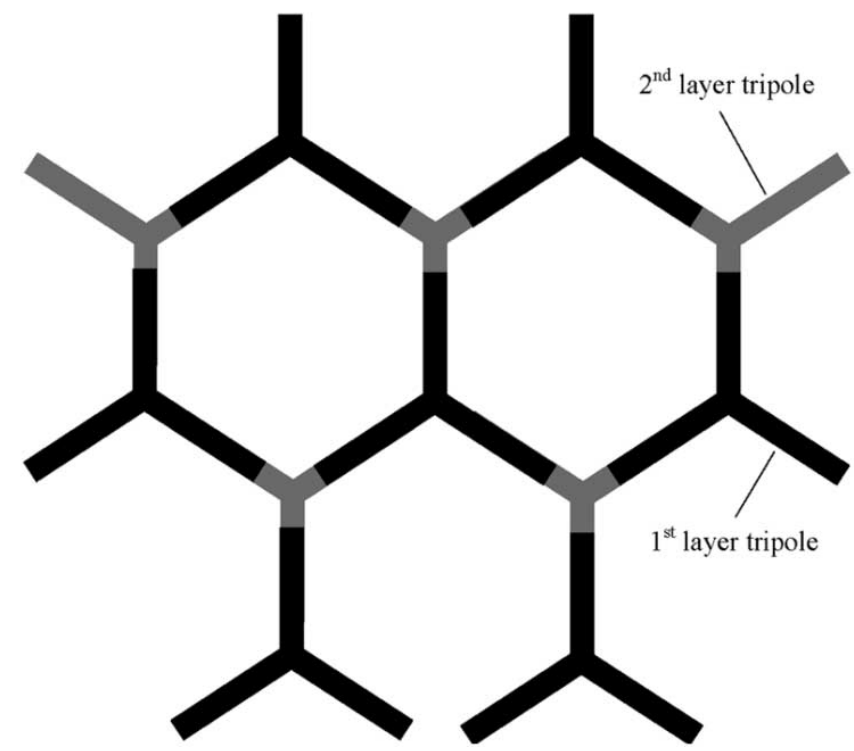

Fig. 6. (a) Geometry of tripole array unit cell, element orientation and reciprocal lattice. (b) Tripole CCMEBG arrangement $\left(0^{\circ}\right.$ orientation).

cut off (passband frequencies), due to the tightening of the radiating fields to the surface. A certain type of surface wave can also exist below and above the band gap, [22]. In addition, the roll-off between passband and stopband has become substantially steep. Due to this steep roll-off, very low transmission values are obtained at the beginning of the stopband, which is not the case with the smoother plane wave responses. The bandwidth of the stopband is quite wide in both cases, with the CCMEBG transmission response having values below $-10 \mathrm{~dB}$ over a wide range of frequencies from 2.5 up to $18 \mathrm{GHz}$, whereas the MEBG response exhibits higher values below 7.4 and above $13.5 \mathrm{GHz}$.

\section{TRIPOLE CCMEBG STRUCTURES}

The dipole is a single polarization element, thus it cannot produce a common band gap when used in a two-dimensional (2-D) EBG design. A tripole element is composed of three dipoles arranged at an angle of $120^{\circ}$ with each other and connected at one end [Fig. 6(a)]. As a more symmetrical 2-D element the tripole can be used in EBG designs which exhibit absolute band gap in the plane of the array for surface wave propagation. In addition, a tripole array under plane wave incidence exhibits a similar band gap for any polarization of the incident field [15]. The tripole element response depends on the contribution of the current on each of the three tripole arms. Thus the angle of symmetry for the tripole element is $30^{\circ}$ which coincides with the angle of the first Brillouin zone of the hexagonal lattice [23]. Therefore, the irreducible Brillouin zone for a tripole array arranged on a hexagonal lattice is $30^{\circ}$ [Fig. 6(a)]. This angle is significantly smaller than the angle of the irreducible Brillouin zone of the dipole array, and is a desirable feature in order to obtain common 2-D band gap.

Tripole arrays in single-layer $\mathrm{MEBG}$ and double-layer CCMEBG configurations are investigated in this section for their band-gap properties when excited by plane as well as surface waves. We have used a tripole array geometry with the following dimensions: $L=5 \mathrm{~mm}, W=0.5 \mathrm{~mm}$, $D_{u}=D_{v}=D=12 \mathrm{~mm}$ [Fig. 6(a)]. The array is printed on a thin dielectric layer of $\varepsilon_{r}=3$ and then mounted on a 


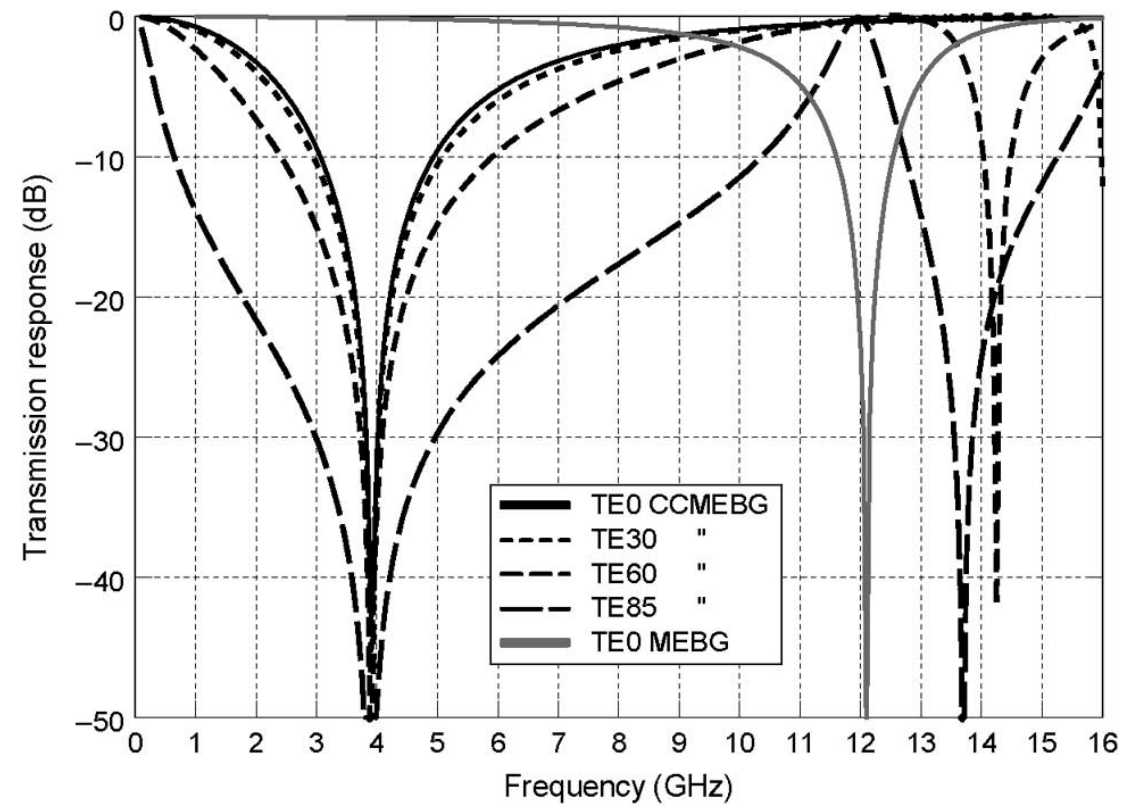

Fig. 7. Simulated transmission response of tripole CCMEBG for different angles of plane wave incidence and tripole MEBG for normal incidence $\left(0^{\circ}\right.$ element orientation).

flat dielectric board similar to the one used with the dipole arrays in order to produce the single-layer tripole MEBG. A double-layer array is formed by printing the tripoles on either side of the thin dielectric layer. The thickness of the thin layer was actually measured $0.15 \pm 0.02 \mathrm{~mm}$, as in the case of the dipole CMMEBG. Following the dipole CCMEBG design, in order to produce maximum coupling between the two layers, we need to place the points of maximum current of the second array in the interelement space of the first one. To achieve that, we rotate the tripole elements of the second layer by $60^{\circ}$ and shift them in the $y$-direction by $D \tan 30^{\circ}=6.928 \mathrm{~mm}$. This arrangement is shown in Fig. 6(b) for $0^{\circ}$ element orientation.

\section{A. Simulations}

The plane wave response of the tripole CCMEBG as well as the single-layer MEBG is simulated using the modal analysis. The simulated transmission coefficients of the tripole MEBG (normal incidence) and CCMEBG (normal and oblique TE incidence) are shown in Fig. 7. The separation distance here was taken $S=0.1 \mathrm{~mm}$. These are results for $0^{\circ}$ orientation of the tripole. The simulations for a $30^{\circ}$ tripole orientation are not shown since they produced similar (although not exactly the same) responses. The resonance in the single-layer MEBG response will be affected by grating lobes due to array periodicity, particularly at high incident angles. The CCMEBG on the other hand exhibits an extremely stable resonance with respect to the angle of incidence (Fig. 7). Grating responses at high angles of incidence appear as nulls in the transmission response at higher frequencies. The resonant frequency has moved from 12.1 (MEBG) to $3.9 \mathrm{GHz}$ (CCMEBG) which corresponds to a relative shift of more than 3:1. The tripole CCMEBG design yields approximately a $\lambda / 15$ resonance, with regards to the length of the tripole arm. Finally, a wider band gap is predicted for the CCMEBG design ( $25 \%$ for the $-10 \mathrm{~dB}$ bandwidth) compared to the MEBG (8.3\%) for normal incidence.

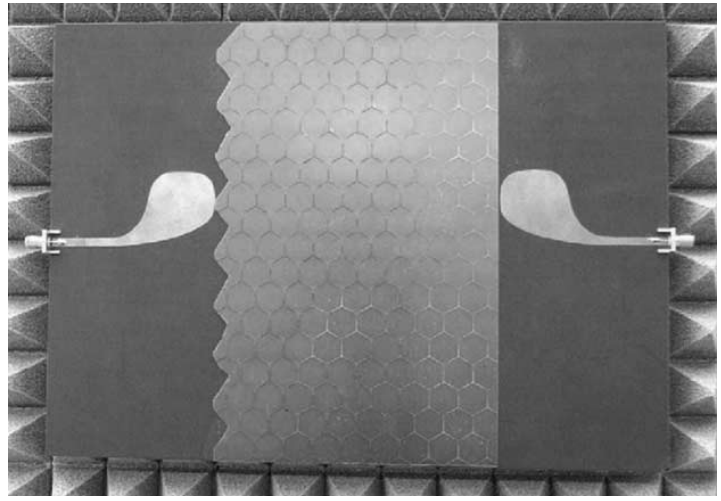

Fig. 8. Photograph of surface wave (SW) measurements configuration with tripole CCMEBG $\left(0^{\circ}\right.$ element orientation).

\section{B. Measurements}

The tripole MEBG and CCMEBG structures have been fabricated as $20-\mathrm{cm}$ square sheets attached on the flat dielectric board of the same size. Plane wave measurements have been carried out for normal and oblique TE incidence using the Vivaldi antennas described in Section III. The configuration shown in Fig. 8 has been used to measure the surface wave response of the arrays. Both element orientations $\left(0\right.$ and $\left.30^{\circ}\right)$ have been investigated and are presented here.

The transmission responses of the MEBG and the CCMEBG design with $0^{\circ}$ element orientation are shown in Fig. 9. The resonance of the MEBG at normal incidence appears at about $11.8 \mathrm{GHz}$. In the CCMEBG design, the resonance at normal incidence has moved to $5.2 \mathrm{GHz}$, which corresponds to more than $2: 1$ frequency shift. This is approximately a $\lambda / 12$ resonance. Moreover, a very good angular stability of the resonance is observed with the CCMEBG array. The CCMEBG design exhibits a 17\% fractional $-10 \mathrm{~dB}$ bandwidth in the stopband compared to a $7.5 \%$ obtained with the MEBG design. The surface wave 
(a)

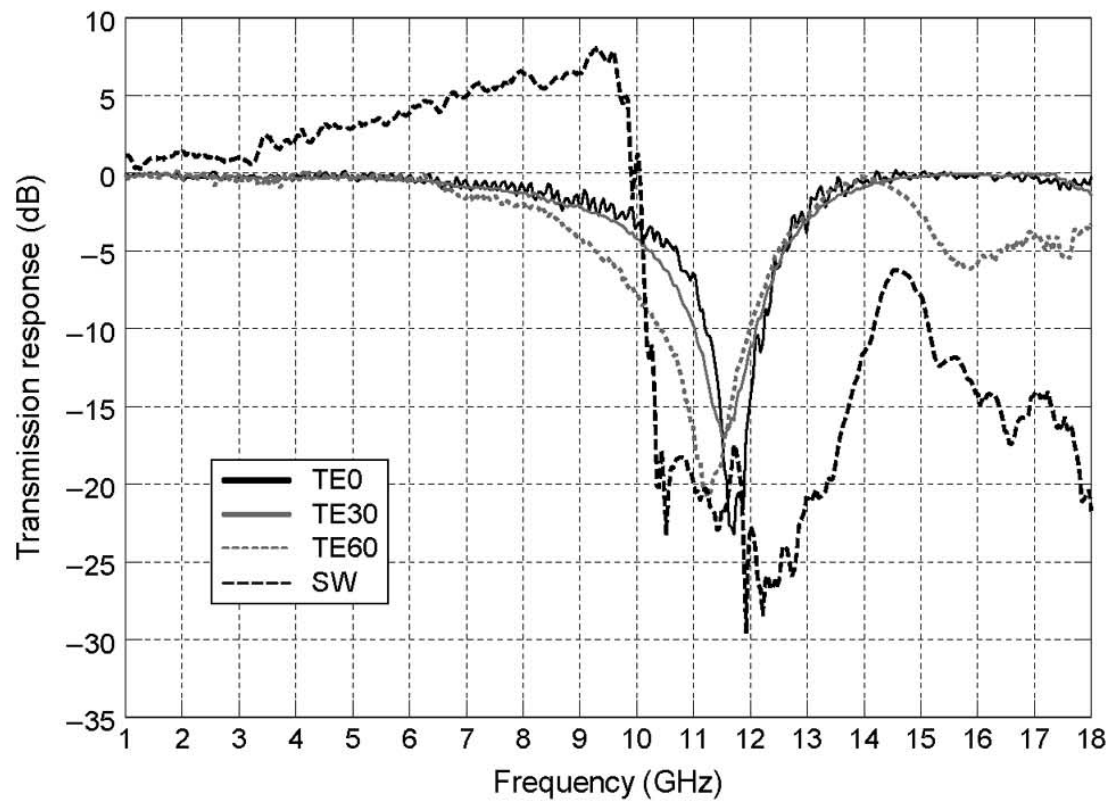

(b)

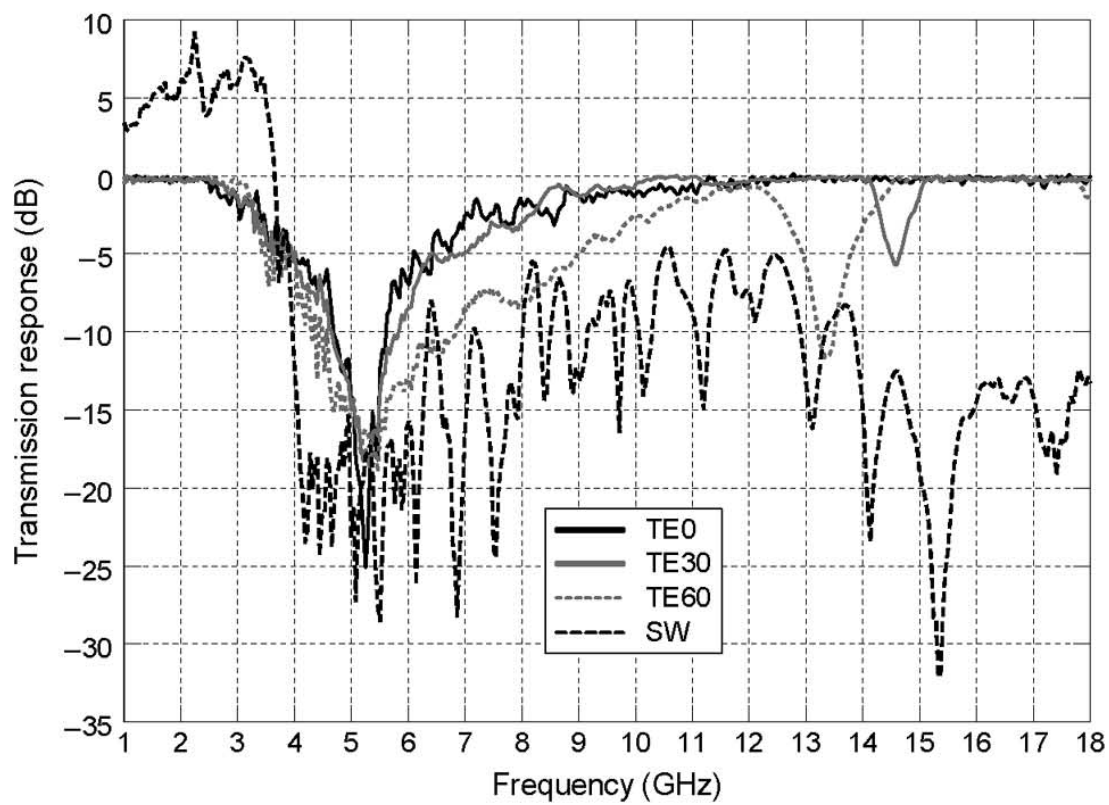

Fig. 9. Measured transmission response of (a) single-layer tripole MEBG. (b) Tripole CCMEBG for TE plane wave and surface wave (SW) incidences ( $0^{\circ}$ element orientation).

responses of both designs exhibit a relative gain of up to about $8 \mathrm{~dB}$ at a wide range of frequencies before the stopband. The transition from passband to stopband is very steep as in the case of the dipole arrays in Section III. The emergence frequency of the stopband for the CCMEBG compared to the MEBG is also shifted by more than $2: 1$, as in the plane wave measurements.

The measurements for $30^{\circ}$ orientation of the tripole are shown in Fig. 10. Comparing with the $0^{\circ}$ orientation, a similar frequency shift is achieved using the CCMEBG design for both plane and surface wave measurements. This confirms the fact that tripole elements are less sensitive to the polarization of the incident field. However, the plane wave responses exhibit slightly wider stopbands $(28 \%-10 \mathrm{~dB}$ fractional bandwidth for the CCMEBG, $9.5 \%$ for the MEBG). Another interesting point is that the surface wave response of the single-layer MEBG shows lower transmission values in the stopband, when compared to the response of the $0^{\circ}$ oriented tripoles, for a wide range of frequencies. The CCMEBG surface wave response however is similar for both orientations.

\section{CONCLUSION}

We have introduced the concept of CCMEBG structures and studied their transmission characteristics for plane wave as well as surface wave excitation. Dipoles has been used initially to implement a CCMEBG design. A significant lowering of the band-gap frequency (4:1) together with a superior angular stability and bandwidth have been predicted using a plane wave 
(a)

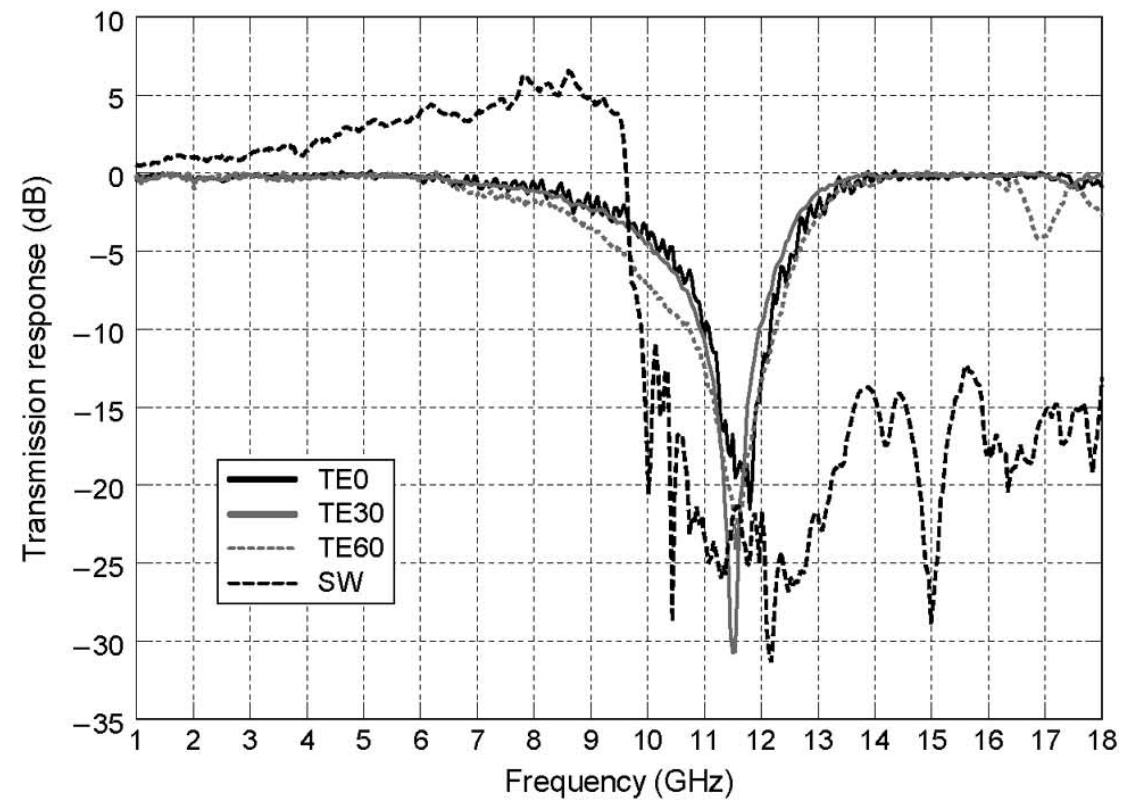

(b)

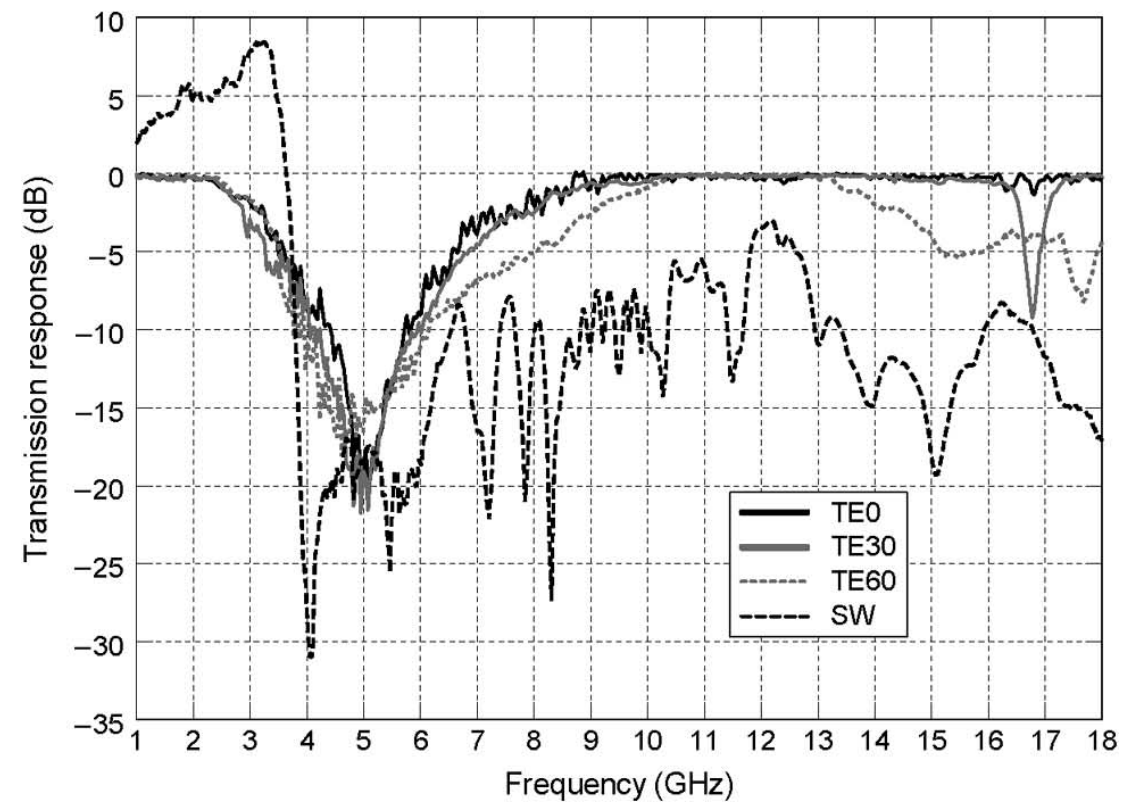

Fig. 10. Measured transmission response of (a) single-layer tripole MEBG. (b) Tripole CCMEBG for TE plane wave and surface wave (SW) incidences (30 ${ }^{\circ}$ element orientation).

modal analysis of the structure. The trends shown in the simulation results have been confirmed with measurements carried out for plane wave as well as surface wave excitation. Despite the discrepancies at low frequencies due to the small size of the arrays, the simulations provided useful design guidelines upon which the construction of the CCMEBG structures was based. For plane wave measurements, a frequency shift of more than 3:1 has been obtained with the dipole CCMEBG, in conjunction with improved angular stability of the resonant frequency. The fractional bandwidth of the stopband also increased by $56 \%$ using the CCMEBG design. A similar shift in the stopband has been obtained during the surface wave measurements. An interesting characteristic of the surface wave response for both single MEBG and double-layer CCMEBG arrays is that a rel- ative gain of up to $8 \mathrm{~dB}$ is obtained in the passband and the transition to the stopband is very steep. Furthermore, the bandwidth of the stopband is wider compared to the one obtained in the plane wave measurements. Tripoles have been used to form CCMEBG structures which are not dependant on the polarization of the incident field. Tripole elements arranged on a hexagonal lattice exhibit common band gap for any incident polarization. The tripole CCMEBG design exhibits a 2:1 frequency shift with respect to the single-layer MEBG. The angular stability of the stopband is improved and the bandwidth is increased. The $30^{\circ}$ element orientation yields a wider bandwidth.

The frequency shift property of CCMEBGs makes these structures very attractive for designs of compact EGB materials with superior angular stability and bandwidth performance. 
Symmetrical 2-D elements can be used in order to obtain common band gaps. Further research is being carried out with regards to the effect of the overall size of the arrays to the band gap. Conformal designs of CCMEBGs could be used as filters for both plane and surface wave propagation.

\section{ACKNOWLEDGMENT}

The authors would like to acknowledge Y. R. Lee for his help and useful discussions.

\section{REFERENCES}

[1] E. Yablonovitch, "Photonic crystals," J. Mod. Opt., vol. 41, pp. 173-194, 1994.

[2] J. D. Joannopoulos, R. D. Meade, and J. N. Winn, Photonic Crystals: Molding the Flow of Light. Princeton, NJ: Princeton Univ. Press, 1995.

[3] IEEE Trans. Microwave Theory Tech.: Special Issue on Electromagnetic Crystal Structures, vol. 47, Nov. 1999.

[4] K. A. McIntosh, O. B. McMahon, and S. Verghese, "Three-dimension metalodielectric photonic crystals incorporating flat metal elements," Microw. Opt. Technol. Lett., vol. 17, no. 3, pp. 153-156, 1998.

[5] R. Gonzalo, P. de Maagt, and M. Sorolla, "Enhanced patch antenna performance by suppressing surface waves using photonic bandgap substrates," IEEE Trans. Microwave Theory Tech., vol. 47, pp. 2131-2138, Nov. 1999.

[6] A. P. Feresidis and J. C. Vardaxoglou, "High gain planar antenna using optimized partially reflective surfaces," Proc. Inst. Elect. Eng. Microwave Antennas Propagat., vol. 148, pp. 345-350, Dec. 2001.

[7] W. M. Merrill, C. A. Kyriazidou, H. F. Contopanagos, and N. G. Alexopoulos, "Electromagnetic scattering from a PBG material excited by an electric line source," IEEE Trans. Microwave Theory Tech., vol. 47, pp. 2105-2114, Nov. 1999.

[8] A. S. Barlevy and Y. Rahmat-Samii, "Characterization of electromagnetic band-gaps composed of multiple periodic tripods with interconnecting vias: Concept, analysis, and design," IEEE Trans. Antennas Propagat., vol. 49, pp. 343-353, Mar. 2001.

[9] Y. R. Lee, A. Charaya, D. S. Lockyer, and J. C. Vardaxoglou, "Dipole and tripole metallodielectric photonic bandgap (MPBG) structures for microwave filter and antenna applications," Proc. Inst. Elect. Eng. Optoelectron., vol. 127, pp. 395-400, Dec. 2000.

[10] B. A. Munk, Frequency Selective Surfaces: Theory and Design. New York: Wiley, 2000

[11] S. W. Schneider and B. A. Munk, "The reflection and transmission properties of 'super dense' surfaces," in Proc. IEEE Int. Conf. Electromagn. Aerospace Applications, Torino, Italy, Sept. 1993, pp. 107-109.

[12] J. C. Vardaxoglou and D. Lockyer, "Modified FSS response from two sided and closely coupled arrays," Electron. Lett., vol. 30, no. 22, pp. 1818-1819, 1994.

[13] D. S. Lockyer and J. C. Vardaxoglou, "Reconfigurable FSS response from two layers of slotted dipole arrays," Electron. Lett., vol. 32, no. 6, pp. 512-513, 1996.

[14] D. S. Lockyer, J. C. Vardaxoglou, and R. A. Simpkin, "Complementary frequency selective surfaces," Proc. Inst. Elect. Eng. Microwave Antennas Propagat., vol. 147, pp. 501-507, Dec. 2000.

[15] J. C. Vardaxoglou, Frequency Selective Surfaces: Analysis and Design. New York: Wiley, 1997

[16] R. Mittra, C. H. Chan, and T. Cwik, "Techniques for analyzing frequency selective surfaces-A review," IEEE Proc., vol. 76, pp. 1593-1615, Dec. 1988.

[17] R. F. Harrington, Field Computation by Moment Methods. New York: MacMillan, 1968

[18] N. V. Shuley, "Higher-order mode interaction in planar periodic structures," Proc. Inst. Elect. Eng. Microwave Antennas Propagat., ser. H, vol. 131, pp. 129-132, 1984.

[19] F. S. Johansson, "Analysis and design of double layer frequency selective surfaces," Proc. Inst. Elect. Eng. Microwave Antennas Propagat., ser. H, vol. 132, pp. 319-325, 1985.

[20] R. Orta, R. Tascone, and R. Zich, "Multiple dielectric loaded perforated screen as frequency selective surfaces," Proc. Inst. Elect. Eng. Microwave Antennas Propagat., ser. H, vol. 135, pp. 75-82, 1988.
[21] E. Gazit, "Improved design of the Vivaldi antenna," Proc. Inst. Elect. Eng. Microwave Antennas Propagat., ser. H, vol. 135, pp. 89-92, 1988.

[22] B. A. Munk, D. S. Janning, J. B. Pryor, and R. J. Marhefka, "Scattering from surface waves on finite FSS," IEEE Trans. Antennas Propagat., vol. 49, pp. 1782-1793, Dec. 2001.

[23] C. Kittel, Introduction to Solid State Physics. New York: Wiley, 1996.

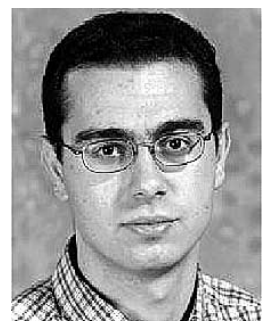

Alexandros P. Feresidis (M'98) was born in Thessaloniki, Greece, in 1975. He received the B.Sc. (Ptychio) degree in physics from Aristotle University of Thessaloniki (AUTH), Greece, in 1997, the M.Sc. (Eng.) degree in radio communications and high-frequency engineering from the University of Leeds, U.K., in 1998, and the Ph.D. degree in electronic and electrical engineering from Loughborough University, U.K., in 2002.

During the first half of 2002, he was a Research Associate with the Wireless Communications Research Group, Department of Electronic and Electrical Engineering, Loughborough University. He is currently a Lecturer in the same department. His research interests include analysis and design of EBG materials and frequency selective surfaces (FSS), periodic structures, high-gain array antennas, base station antennas, evolutionary computation techniques in electromagnetics, and microwave circuits.

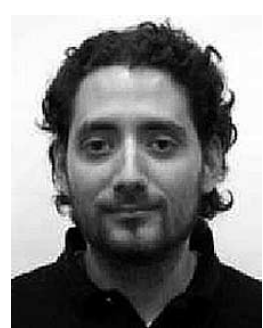

George Apostolopoulos (S'03) was born in San Felix, Venezuela, in 1977. He received the B.Eng. degree in telecommunication engineering from Essex University, U.K., in 2000 and the M.Sc. degree in digital communication systems for antennas and mobile applications from Loughborough University, U.K., in 2001, where he is currently working toward the Ph.D. degree in electrical and electronic engineering. His doctoral research concerns metallodielectric EBG structured marerials.

His research interests include electromagnetic band gap structures, base station antennas, and frequency selective surfaces.

Nikolaos Serfas, photograph and biography not available at the time of publication.

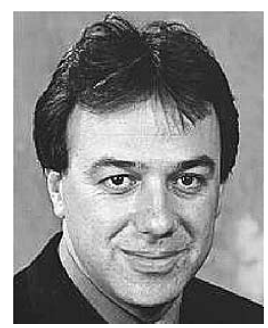

John (Yiannis) C. Vardaxoglou (M'87) received the B.Sc. degree in mathematics (mathematical physics) and the Ph.D. degree from the University of Kent at Canterbury, U.K., in 1981 and 1985, respectively.

In January 1988, he was appointed Lecturer in Communications with the Department of Electronic and Electrical Engineering, Loughborough University of Technology, U.K. He was promoted to the position of Senior Lecturer in January 1992. In 1998, he was appointed Professor of Wireless Communications. He established the Antennas and Microwaves Research Group at Loughborough University and he heads the Centre for Mobile Communications Research (CMCR). He has been active in the area of electromagnetic modeling and applications of frequency selective surfaces (FSS). His current research interests include wireless communication networks, array antennas, FSS, radomes, leaky-wave resonant antennas, optical control of microwaves and devices, periodic surfaces and EBG/PBG materials, and mobile telephone antennas. His contribution to the CMCR lies in the analysis and design of small-loaded antennas for mobile telephony. He has served as a Consultant to various industries in the U.K. and abroad. He has published more than 120 refereed journals and conference proceeding papers and has written a research monograph on Frequency Selective Surfaces. 